\title{
Induction and Coexpression of Latent Transforming Growth Factor $\beta$-Binding Protein-1 and Fibrillin-1 in Experimental Glomerulonephritis
}

\author{
Markus Porst ${ }^{\mathrm{a}}$ Christoph Daniel $^{\mathrm{b}}$ Christian Plank $^{\mathrm{a}}$ Harald O. Schocklmann ${ }^{\mathrm{c}}$ \\ Dieter P. Reinhardt ${ }^{d}$ Andrea Hartner ${ }^{a}$ \\ ${ }^{a}$ Children's Hospital and bepartment of Medicine IV, University of Erlangen-Nuremberg, Erlangen, \\ ${ }^{\mathrm{c}}$ Department of Nephrology, University of Kiel, Kiel, Germany; ${ }^{\mathrm{d}}$ Department of Anatomy and Cell Biology and \\ Faculty of Dentistry, McGill University, Montreal, Canada
}

\section{Key Words}

Glomerulus · Mesangial cells · Extracellular matrix •

Microfibrillar proteins · Transforming growth factor- $\beta$ •

Anti-Thy1.1 nephritis

\begin{abstract}
Background: Latent transforming growth factor- $\beta$-binding protein 1 (LTBP-1) and fibrillin-1 were shown to colocalize and interact in the extracellular matrix of the skin and vasculature. This interaction may regulate transforming growth factor- $\beta$ (TGF- $\beta$ ) activity. TGF- $\beta$ is an important progression factor for glomerular diseases. We hypothesized that LTBP-1 and fibrillin-1 are coexpressed in the glomerulus and upregulated during glomerulonephritis. Methods: Acute anti-Thy1.1 glomerulonephritis was induced with a single intravenous injection $(1 \mathrm{mg} / \mathrm{kg}$ body weight) of a monoclonal anti-Thy1.1 antibody in rats. Real-time RT-PCR and immunohistochemical analyses for LTBP-1 and fibrillin-1 were performed. Results: Induction of glomerular LTBP-1 mRNA was detected on day 2 of disease, while mRNA for fibrillin-1 was already upregulated 1 day after induction of disease. Both LTBP-
\end{abstract}

1 and fibrillin-1 showed a mesangial distribution. An expansion of the LTBP-1 and fibrillin-1-positive mesangial area was seen on day 6 of disease, when transient matrix accumulation was most prominent. On day 12 of disease, glomerular LTBP-1 and fibrillin-1 immunoreactivities had returned to control levels. In serial sections, some colocalization of LTBP-1 and fibrillin-1 was detected in control as well as in nephritic glomeruli. Conclusion: Mesangial expression of LTBP-1 and fibrillin-1 is induced early in experimental nephritis and LTBP-1 and fibrillin-1 are partially colocalized in the nephritic glomerulus. An interaction of these molecules could stabilize latent TGF- $\beta$ complexes and thus attenuate the activation of TGF- $\beta$ during this self-limited glomerular disease.

Copyright $(2006$ S. Karger AG, Basel

Latent transforming growth factor- $\beta$-binding protein 1 (LTBP-1) regulates secretion and activation of transforming growth factor- $\beta$ (TGF- $\beta$ ) isoforms [1] and also plays a role as a structural component of fibrillin-rich microfibrils in the extracellular matrix of the vasculature or

\section{KARGER \\ Fax +4161306 1234 E-Mail karger@karger.ch} www.karger.com (c) 2006 S. Karger AG, Basel $1660-2129 / 06 / 1024-0099 \$ 23.50 / 0$

Accessible online at: www.karger.com/nee
Andrea Hartner, PhD

Klinik für Kinder und Jugendliche

Loschgestrasse 15, DE-91054 Erlangen (Germany)

Tel. +49 91318536873, Fax +49 91318539202

E-Mail andrea.hartner@rzmail.uni-erlangen.de 
of the eye [2]. LTBP-1 and fibrillin-1 are highly homologous molecules and have been shown to colocalize and interact in the extracellular matrix of these tissues [3]. The interaction of LTBP-1 and fibrillin- 1 seems to be important for the stabilization of latent TGF- $\beta$ complexes in the extracellular matrix [4]. In fibrillin-1-deficient mice, activation of TGF- $\beta$ was recently described [5]. It is conceivable that the loss of fibrillin-1 destabilizes latent TGF$\beta$ complexes. In earlier studies, we have shown that glomerular mesangial cells express and secrete both LTBP-1 and fibrillin-1 in vitro [6]. Further, we and others have demonstrated an induction of TGF- $\beta$ isoforms during experimental anti-Thy1.1 glomerulonephritis, which is characterized by transient mesangial hypercellularity and mesangial matrix expansion $[7,8]$. We hypothesized that LTBP-1 and fibrillin-1 are coexpressed in the mesangial matrix and are upregulated during acute anti-Thy 1.1 glomerulonephritis.

\section{Methods}

\section{Experimental Disease}

Male Sprague-Dawley rats (150-200 g) were obtained from Charles River Deutschland (Sulzfeld, Germany). Anti-Thy1.1 nephritis was induced by a single intravenous injection of $1 \mathrm{mg} / \mathrm{kg}$ body weight of anti-Thy1.1 antibody (ER4, Antibody Solutions, Palo Alto, Calif., USA) into the tail vein. Controls received solvent only. Four animals in each group were sacrificed on days $0,1,2$, 3 and 5 (for mRNA analysis); and on days 0,6 and 12 (for immunohistochemistry) after induction of nephritis. The kidneys were decapsulated and one kidney was used for isolation of glomeruli. The second kidney was embedded in paraffin or processed for preparation of cryostat sections as described previously [7, 9]. For glomerular RNA isolation, glomeruli were isolated as described [7].

\section{Immunohistochemistry}

Staining of cryostat or paraffin sections was performed as described elsewhere [7]. The primary rabbit antibody to fibrillin-1 [10] was used at a dilution of 1:500. The primary antibody to LTBP1 (1:250) was obtained from Pharmingen (Hamburg, Germany). CY3-labelled anti-rabbit IgG (DAKO Diagnostica, Hamburg, Germany) or secondary horseradish peroxidase-conjugated anti-rabbit IgG (Vectastain, Vector Lab, Burlingame, Calif., USA) were used as secondary antibodies. Glomerular fibrillin-1 and LTBP-1 staining was evaluated by densitometry using Metaview software. Staining was measured in every third glomerulus per cross-section of frozen kidney and was expressed as a percentage of stained area per glomerular tuft.

\section{Isolation of mRNA and Real-Time PCR}

To evaluate mRNA expression levels, total RNA was obtained from isolated glomeruli with RNeasy Mini columns (Quiagen, Hilden, Germany). Reverse transcription reactions and PCR were performed according to the manufacturer's instructions (Applied
Biosystems, Weiterstadt, Germany) using SYBR green reagents. The relative amount of specific $m R N A$ was normalized with respect to $18 \mathrm{~S}$ rRNA. Primers used for amplification of $18 \mathrm{~S}$ cDNA were forward 5'TTGATTAAGTCCCTGCCCTTTGT3' and reverse 5'CGATCCGAGGGCCTCACTA3'. For amplification of the rat fibrillin-1 cDNA, the forward primer was 5'TGCTCTGAAAGGACCCAATGT3' and the reverse primer was 5'CGGGACAACAGTATGCGTTATAAC3'. For amplification of the rat LTBP-1 cDNA the forward primer was 5'CGGATCCCCCTATGATCTCA 3 ' and the reverse primer was 5'TGACGAGGCGGTAGCAGG3'. All samples were analyzed in triplicate.

\section{Statistical Analyses}

Two-way analysis of variance (ANOVA), followed by post hoc Newman-Keuls test with adjustment for multiple comparisons, was used to test significance of differences between groups. A p value of $<0.05$ was considered significant. The procedures were carried out using SPSS software (SPSS Inc., Chicago, Ill., USA). Values are displayed as means \pm standard error of the mean (SEM) for evaluation of fibrillin-1 and LTBP-1 mRNA expression and protein expansion.

\section{Results}

mRNA expression of fibrillin-1 and LTBP-1 was monitored from day 1 to day 5 of disease. Induction of antiThy 1.1 glomerulonephritis led to an increase in fibrillin1 and LTBP-1 mRNA levels after 1 or 2 days, respectively (fig. 1A, B). Fibrillin-1 mRNA expression was increased fourfold by day 5, while LTBP-1 mRNA expression reached a twofold maximal increase on day 3 of disease (fig. 1). Fibrillin-1 and LTBP-1 protein deposition in the glomerular extracellular matrix was followed until day 12 . In healthy glomeruli, fibrillin-1 and LTBP-1 were detected in areas consistent with a mesangial distribution (fig. 2A, B).

A marked expansion of fibrillin-1 and LTBP-1 immunoreactivity occurred on day 6 of disease (fig. 2C, D) while on day 12 of disease the distribution of fibrillin-1 and LTBP-1 protein was comparable to that of healthy control glomeruli (fig. 2E, F). Densitometric evaluation of glomerular fibrillin-1 staining during anti-Thy 1.1 glomerulonephritis revealed a transient increase on day 6 $(27.4 \pm 2.2 \%$ stained area/glomerulus on day 6 vs. 12.5 $\pm 0.9 \%$ in controls, $\mathrm{p}<0.05, \mathrm{n}=7$ per group), which corresponds to the stage of maximal glomerular hypercellularity and glomerulosclerosis [7]. By day 12 of this transient form of anti-Thy 1.1 glomerulonephritis, glomeruli appear to be normal [7]. On day 12, densitometric evaluation showed that fibrillin-1 levels had almost reached the values of healthy glomeruli (16.9 $\pm 1.4 \%$ stained area/ glomerulus, $n=6$ ). Similar results were obtained for 
LTBP-1 protein $(36.0 \pm 3.4 \%$ stained area/glomerulus on day 6 , vs. $16.7 \pm 1.1 \%$ in controls and $19.5 \pm 1.4 \%$ on day 12 of disease, $p<0.05$ ).

To test whether fibrillin-1 and LTBP-1 are colocalized, consecutive cryosections were stained for fibrillin-1 or LTBP-1 (fig. 3). In healthy glomeruli, staining for fibrillin-1 was partially colocalized with the LTBP-1 staining (fig. 3A, B). On day 6 of disease, some colocalization of fibrillin-1 and LTBP-1 was also detected (fig. 3C, D).

\section{Discussion}

Glomerular LTBP-1 and fibrillin-1 protein expression has been described previously in humans and rats [6]. We now demonstrate an induction of glomerular LTBP1 and fibrillin-1 mRNA expression and protein expansion during experimental glomerulonephritis. mRNA expression of these molecules is induced soon after onset of the disease. Our results further reveal a colocalization, at least partial, of LTBP-1 and fibrillin-1 in the mesangial matrix.

The function of these two molecules in the glomerulus is not yet clear. Excessive deposition of LTBP-1 was detected in more severe forms of human IgA nephropathy [11]. Studies in cultured mesangial cells suggested that the activity of LTBP-1 contributes to the upregulation of mRNA expression of matrix molecules following mechanical stress [12]. Thus it seems possible that LTBP-1 is a progression factor, at least in chronic glomerular disease. A dual role for LTBP-1 is proposed [2]. On one hand, it serves as a structural matrix protein. On the other hand, it contributes to storage of latent TGF- $\beta$ [2] and could thus regulate the activity of TGF- $\beta$ by orchestrating efficient secretion and correct folding of growth factors of the TGF- $\beta$ family [1].

In many tissues, fibrillin- 1 is a component of elastic fibers and acts as a template for elastin deposition [13]. In the mesangial matrix of the glomerulus, however, no elastin expression was found [6]. Thus, it remains unclear, as to whether fibrillin-1 has an elastic function in the glomerulus. Induction of fibrillin-1 was detected in kidney tissue in pressure-induced renal disease [14], where it seemed to serve a protective role for the structure and function of the kidney. In this disease, most morphological alterations are seen in the tubulo-interstitium of the renal cortex (where elastin is abundant), and not in the glomeruli.

The coexpression of LTBP-1 and fibrillin-1 in the mesangial matrix and the partial overlap of the localization of both molecules argue for a cross-talk between LTBP-1

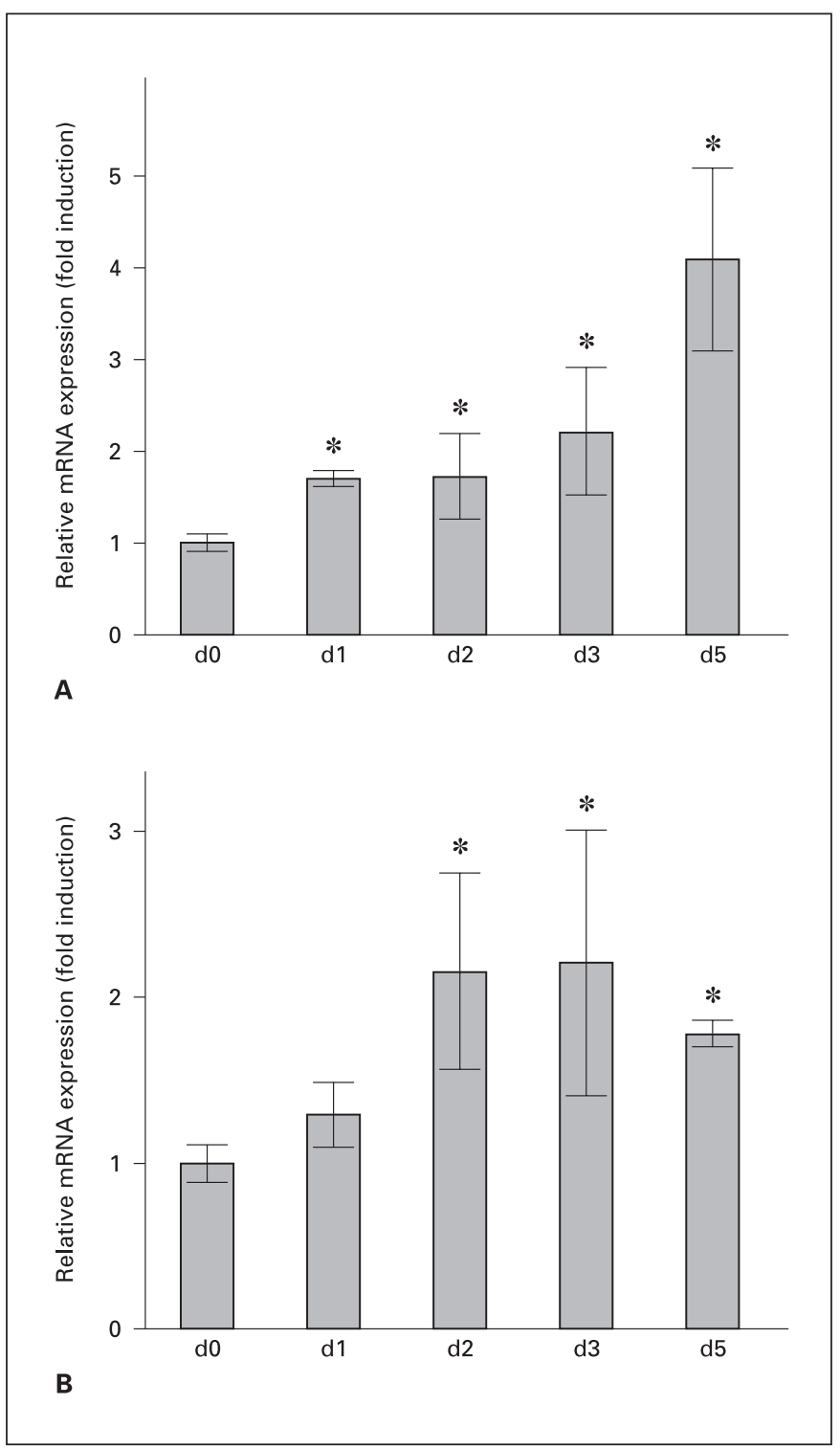

Fig. 1. Induction of fibrillin-1 (A) and LTBP-1 (B) mRNA expression in isolated glomeruli in the early phase of Thy 1.1 glomerulonephritis as assessed by real-time RT-PCR. Data are means \pm standard error of the mean. ${ }^{*} \mathrm{p}<0.05$ Thy 1.1 nephritic rats vs. controls. $\mathrm{d}=$ Days.

and fibrillin-1 in the glomerulus, although this does not necessarily imply a functional interaction. Isogai et al. [3] performed binding studies demonstrating that LTBP-1 and fibrillin-1 are able to interact on the molecular level. Some studies showing coexpression of LTBP-1 and fibrillin-1 exist: in a porcine model of coronary angioplasty, induction of LTBP-1 and fibrillin-1 expression and colocalization of both molecules were found in the neointima 
Fig. 2. Immunohistochemical detection of fibrillin-1 (A, C, E cryosections) and LTBP1 (B, D, F sections of paraffin-embedded kidney tissue) in glomeruli of control rats (A, B), on day 6 after onset of glomerulonephritis (C, D) and on day 12 after onset of glomerulonephritis (E, F). Magnification $\times 400$.
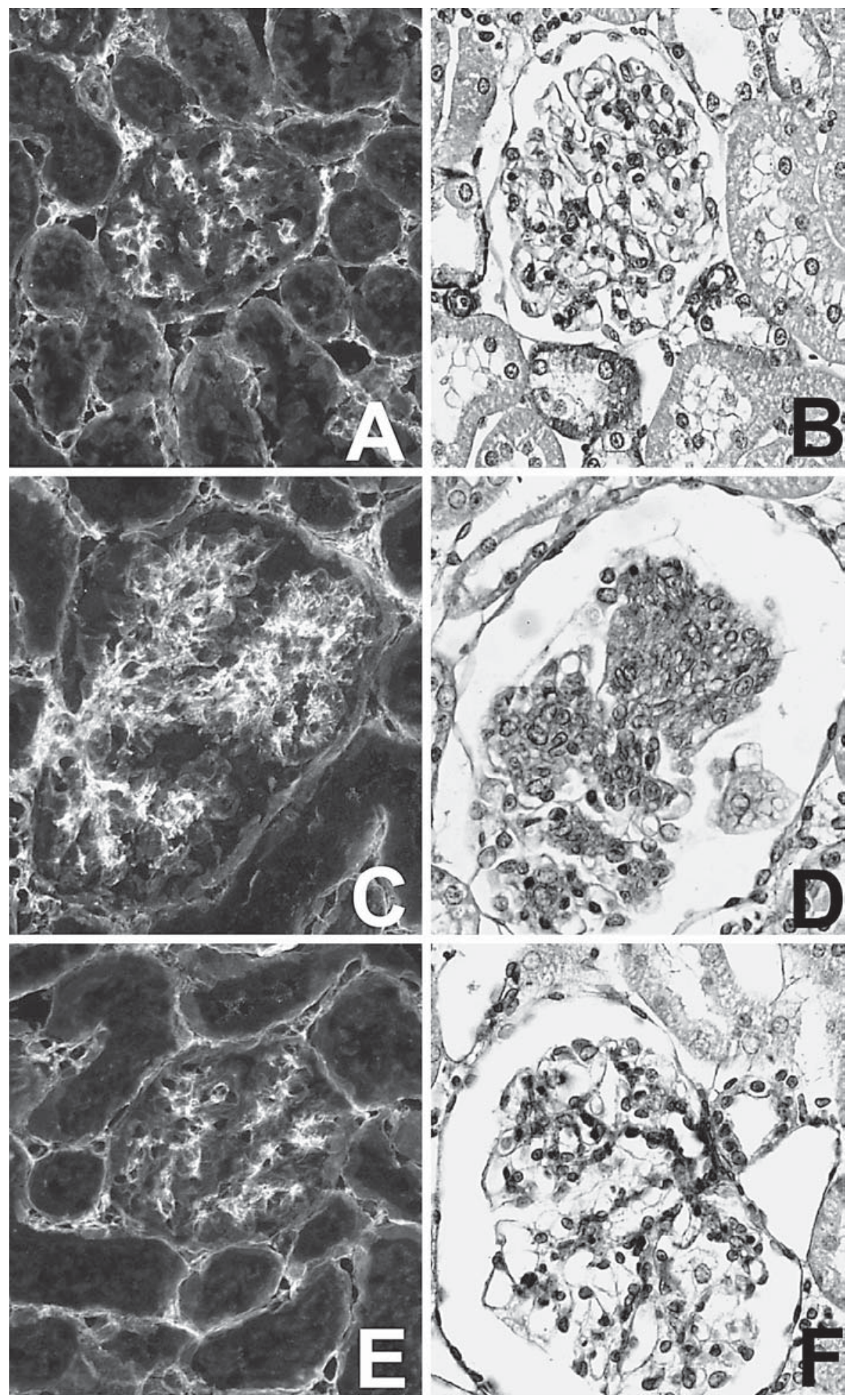
Fig. 3. Immunostaining for fibrillin-1 $(\mathbf{A}, \mathbf{C})$ and LTBP-1 (B, D) in consecutive cryosections of control kidneys (A, B) or nephritic kidneys (C, D) on day 6 after onset of glomerulonephritis. White arrowheads mark examples of colocalization of fibrillin- 1 and LTBP-1 staining. Magnification $\times 400$.
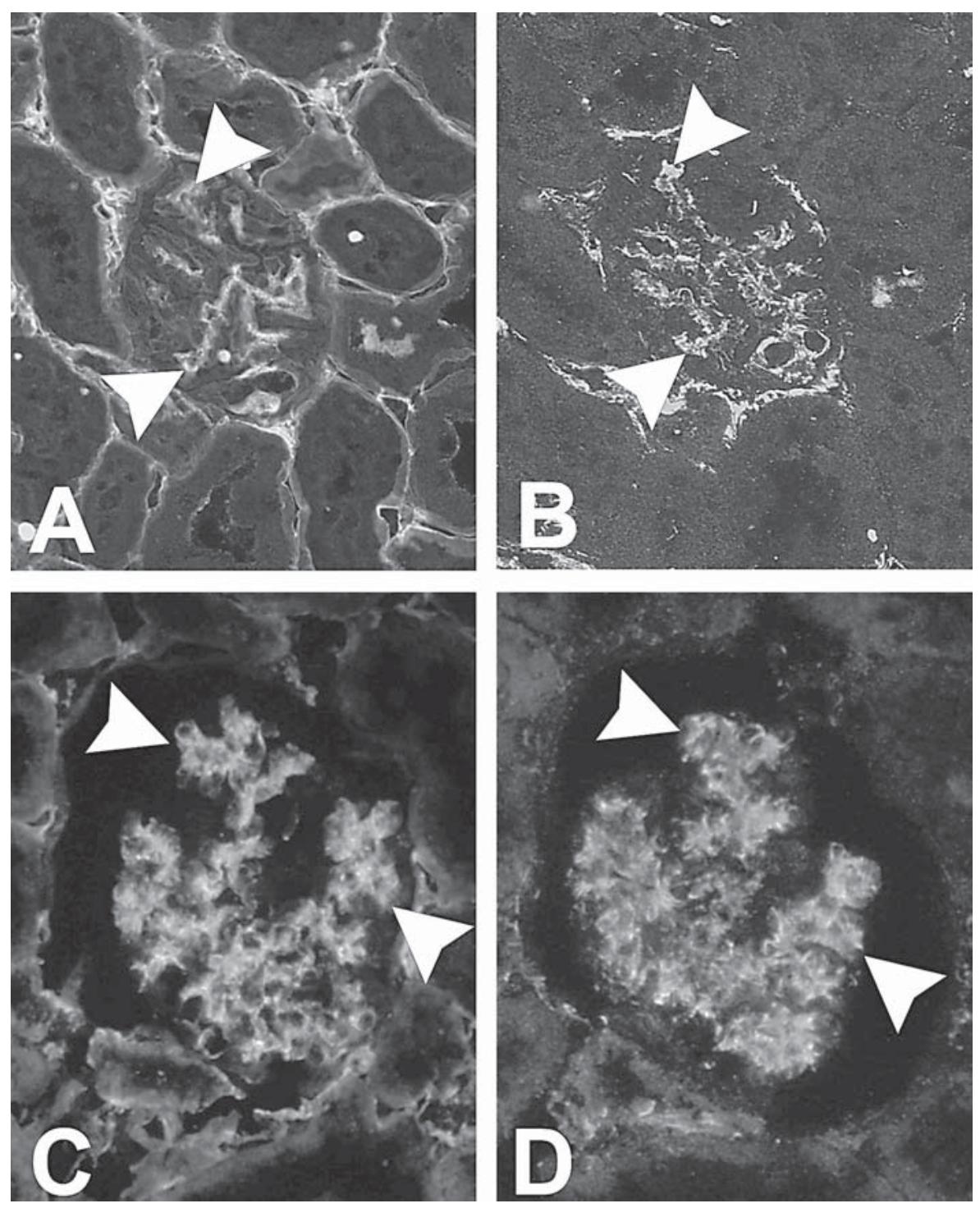

and adventitia [15]. Colocalization of LTBP-1 and fibrillin-1 was also described in pseudo-exfoliation syndrome of the eye [16] and in capsular opacification [17]. Here, lens epithelial cells upregulate LTBP-1 and fibrillin-1 during postoperative healing. Our findings in the glomerulus extend that obtained in other tissues to the renal mesangium and glomerular disease. Whether or not the induction of LTBP-1 and fibrillin-1 has a beneficial effect for the course of anti-Thy1.1 glomerulonephritis is still unclear, but recent data suggest that the interaction of LTBP1 and fibrillin-1 plays an important role in the regulation of TGF- $\beta$ activation $[4,5]$.

Mice deficient in fibrillin-1 were developed as a model to study the pathology of Marfan syndrome [5]. In these mice, a dysregulation of TGF- $\beta$ activation which contributed to the pathogenesis of typical features of the Marfan syndrome was found [4]. Thus, it seems conceivable that an impaired interaction between LTBP-1 and fibrillin-1 in fibrillin-1-deficient mice leads to abnormal activation and signaling of TGF- $\beta$. Given the fact that TGF- $\beta$ s are also transiently upregulated in acute anti-Thy 1.1 glomerulonephritis [7], one could speculate that in this model excessive TGF- $\beta$ activity is scavenged by LTBP- 1 and fibrillin-1 interactions, and this results in a self-limited pathological process in the affected glomeruli and a progression of the disease to a chronic state may be prevented. 


\section{Acknowledgements}

The expert technical assistance of Miroslava KupraszewiczHutzler and Rainer Wachtveitl is gratefully acknowledged. The authors thank Dr. Richard Carr for critically reading the manu- script. This study was supported by a grant from the Deutsche Forschungsgemeinschaft, Bonn, Germany (STE 196/4-1), an ELAN grant from the Faculty of Medicine, University of Erlangen-Nürnberg, and a grant from the Canadian Institutes of Health Research (MOP-68836).

\section{References}

1 Saharinen J, Hyytiainen M, Taipale J, KeskiOja J: Latent transforming growth factor-beta binding proteins (LTBPs) - structural extracellular matrix proteins for targeting TGF-beta action. Cytokine Growth Factor Rev 1999;10: 99-117.

2 Dallas SL, Miyazono K, Skerry TM, Mundy GR, Bonewald LF: Dual role for the latent transforming growth factor-beta binding protein in storage of latent TGF-beta in the extracellular matrix and as a structural matrix protein. J Cell Biol 1995;131:539-549.

3 Isogai Z, Ono RN, Ushiro S, Keene DR, Chen Y, Mazzieri R, Charbonneau NL, Reinhardt DP, Rifkin DB, Sakai LY: Latent transforming growth factor beta-binding protein 1 interacts with fibrillin and is a microfibril-associated protein. J Biol Chem 2003;278: 2750-2757.

$4 \mathrm{Ng} \mathrm{CM}$, Cheng A, Myers LA, Martinez-Murillo F, Jie C, Bedja D, Gabrielson KL, Hausladen JM, Mecham RP, Judge DP, Dietz HC: TGF-beta-dependent pathogenesis of mitral valve prolapse in a mouse model of Marfan syndrome. J Clin Invest 2004;114:15861592.

5 Neptune ER, Frischmeyer PA, Arking DE, Myers L, Bunton TE, Gayraud B, Ramirez F, Sakai LY, Dietz HC: Dysregulation of TGFbeta activation contributes to pathogenesis in Marfan syndrome. Nat Genet 2003;33:407411.
6 Sterzel RB, Hartner A, Schlotzer-Schrehardt U, Voit S, Hausknecht B, Doliana R, Colombatti A, Gibson MA, Braghetta P, Bressan GM: Elastic fiber proteins in the glomerular mesangium in vivo and in cell culture. Kidney Int 2000;58:1588-1602.

7 Hartner A, Hilgers KF, Bitzer M, Veelken R, Schocklmann HO: Dynamic expression patterns of transforming growth factor-beta(2) and transforming growth factor-beta receptors in experimental glomerulonephritis. J Mol Med 2003;81:32-42.

8 Minto AW, Wilson HM, Rees AJ, Quigg RJ, Brown PA: Selective expression of TGF-beta2 and TGF-beta3 isoforms in early mesangioproliferative glomerulonephritis. Nephron Exp Nephrol 2004;96:e111-e118.

9 Hartner A, Cordasic N, Klanke B, Muller U, Sterzel RB, Hilgers KF: The alpha8 integrin chain affords mechanical stability to the glomerular capillary tuft in hypertensive glomerular disease. Am J Pathol 2002;160:861-867.

10 Tiedemann K, Batge B, Muller PK, Reinhardt DP: Interactions of fibrillin-1 with heparin/heparan sulfate, implications for microfibrillar assembly. J Biol Chem 2001;276:36035-36042.

11 Wada T, Hamakawa S, Hori Y, Kaname S, Shimizu S, Kurokawa K, Katoh T: Immunohistochemical localization of latent transforming growth factor-beta binding protein in IgA nephropathy. Kidney Int Suppl 1997;63:S182S184.
12 Hori Y, Katoh T, Hirakata M, Joki N, Kaname S, Fukagawa M, Okuda T, Ohashi H, Fujita T, Miyazono K, Kurokawa K: Anti-latent TGFbeta binding protein-1 antibody or synthetic oligopeptides inhibit extracellular matrix expression induced by stretch in cultured rat mesangial cells. Kidney Int 1998;53:1616-1625.

13 Kielty CM, Wess TJ, Haston L, Ashworth JL, Sherratt MJ, Shuttleworth CA: Fibrillin-rich microfibrils: elastic biopolymers of the extracellular matrix. J Muscle Res Cell Motil 2002; 23:581-596.

14 Schaefer L, Mihalik D, Babelova A, Krzyzankova M, Grone HJ, Iozzo RV, Young MF, Seidler DG, Lin G, Reinhardt DP, Schaefer RM: Regulation of fibrillin-1 by biglycan and decorin is important for tissue preservation in the kidney during pressure-induced injury. Am J Pathol 2004; 165:383-396.

15 Sinha S, Heagerty AM, Shuttleworth CA, Kielty CM: Expression of latent TGF-beta binding proteins and association with TGF-beta 1 and fibrillin-1 following arterial injury. Cardiovasc Res 2002;53:971-983.

16 Schlotzer-Schrehardt U, Zenkel M, Kuchle M, Sakai LY, Naumann GO: Role of transforming growth factor-beta 1 and its latent form binding protein in pseudoexfoliation syndrome. Exp Eye Res 2001;73:765-780.

17 Saika S, Miyamoto T, Tanaka T, Ishida I, Ohnishi Y, Ooshima A: Latent TGFbeta binding protein-1 and fibrillin-1 in human capsular opacification and in cultured lens epithelial cells. Br J Ophthalmol 2001;85:1362-1366. 